\title{
Consuming alcohol as part of a meal is associated with lower risk of Coronary Heart Disease in a UK cohort
}

\author{
M. A. H. Lentjes ${ }^{1}$, A. A. Mulligan ${ }^{1}$, A. P. Khawaja ${ }^{1}$, A. Bhaniani ${ }^{1}$, R. N. Luben ${ }^{1}$ \\ and K. T. Khaw \\ ${ }^{1}$ University of Cambridge, Department of Public Health \& Primary Care, Cambridge CBI $8 R N$ and ${ }^{2}$ University of \\ Cambridge, Clinical Gerontology Unit, Addenbrooke's Hospital, Cambridge CB2 $2 Q Q$
}

Moderate alcohol intake has been found to be protective for coronary heart disease (CHD) in many population-based studies ${ }^{(1)}$. In the European Prospective Investigation into Cancer and Nutrition (EPIC), alcohol intake patterns vary widely across participating centres $^{(2,3)}$ and it remains uncertain how these patterns relate to CHD risk. We assessed how 'with meal' and 'outside meal' alcohol consumption were associated with CHD incidence in EPIC-Norfolk.

Participants completed a health and lifestyle questionnaire and attended a health examination between 1993 and 1998 $(n=25,639)$. Trained nurses gave instructions regarding 7-day diet diary (7dDD) completion. Only participants who returned a complete $7 \mathrm{dDD}$, who had complete covariable data and who did not report a history of stroke, heart attack or cancer at baseline were included $(n=20,274)$. Alcohol intake was compartmentalised into 'with meal' when consumed with any of the following foods: meat, fish, eggs, potatoes, vegetables, cereals or soup; or, 'outside meal' if none of these foods were consumed at time of alcohol consumption. The recommended amount of alcoholic beverages (units/d) were used to create a 'moderate' ( $\leqslant 2.5$ for women; $\leqslant 3.5$ for men) and 'high consumer' group ( $>2.5$ and $>3.5$ respectively); because of unavoidable misclassification in identifying non-alcohol consumers in a $7 \mathrm{dDD}$, we considered this group together with participants reporting an alcohol intake of $<0.5$ unit/d into one "none/very low' category. Cox regression was used to assess the relative risk (hazard ratio, HR) of total and compartmentalised alcohol for CHD (2712 events over a median follow-up of 12 years), adjusted for sex, age, body mass index, smoking, education, social class, total energy intake and physical activity.

Among moderate and high consumers, the proportion of alcohol taken 'with meal' was similar $(P=0 \cdot 38)$. Total and 'with meal' alcohol intake were negatively associated with CHD; however, 'outside meal' alcohol consumed above the recommended intake was associated with increased CHD. As a third model, which contained moderate and high consumers only $(n=11,340$, 1387 events), we substituted 1 unit of 'outside meal' for 'with meal' alcohol whilst keeping total alcohol constant. 'With meal' alcohol was associated

\begin{tabular}{|c|c|c|c|c|c|c|c|c|}
\hline & & \multicolumn{7}{|c|}{ Categories of alcohol intake } \\
\hline \multirow{2}{*}{\multicolumn{2}{|c|}{$\begin{array}{l}\text { Models maximally } \\
\text { adjusted }\end{array}$}} & \multirow{2}{*}{$\begin{array}{l}\text { None/very low } \\
\text { HR }\end{array}$} & \multicolumn{3}{|c|}{ Moderate } & \multicolumn{3}{|c|}{ High } \\
\hline & & & HR & $95 \% \mathrm{CI}$ & $P$ & HR & $95 \% \mathrm{CI}$ & $P$ \\
\hline 1 & Total alcohol & 1.00 (Ref) & $0 \cdot 88$ & $0 \cdot 81,0 \cdot 96$ & 0.004 & 0.89 & $0 \cdot 79,1 \cdot 00$ & $0 \cdot 05$ \\
\hline \multirow[t]{2}{*}{2} & With meal & 1.00 (Ref) & $0 \cdot 83$ & $0 \cdot 76,0.91$ & $<0.001$ & 0.75 & $0.62,0.90$ & 0.002 \\
\hline & Outside meal & 1.00 (Ref) & 1.00 & $0.91,1.09$ & 0.98 & 1.29 & $1.09,1.53$ & 0.003 \\
\hline
\end{tabular}

with a lower CHD risk of $0 \cdot 91$ (95\% CI: 0.87, 0.95).

In this UK cohort, alcohol consumed with a meal was associated with lower CHD risk, whereas high consumption of 'outside meal' alcohol was associated with increased CHD risk. Possible explanations include different choices of alcoholic beverages and/or foods, portion of alcohol per drinking occasion and differences in the time it takes to metabolise alcohol.

1. Ronksley PE, Brien SE, Turner BJ, et al. (2011) BMJ 342, d671.

2. Sieri S, Krogh V, Saieva C, et al. (2009) Eur. J. Clin. Nutr. 63 Suppl 4, S81-100.

3. Sieri S, Agudo a, Kesse E, et al. (2002) Public Health Nutr. 5, 1287-96. 